\title{
Preface: Special Session SpS5 The IR view of massive stars: the main sequence and beyond
}

Though multiwavelength astronomy was born over fifty years ago, the wide-spread use of multiwavelength diagnostics is a more recent phenomenon. Even in the last decade, astronomers continued to rely on the optical domain for the bulk of their analysis. However, this is certain to change, as most of the current and future instruments are increasingly dedicated to observations in the infrared, from the near- to the far-infrared bands.

While the infrared domain is well established in research on low-mass stars, especially the very low-mass ones, the enormous potential for the study and analysis of infrared emission from high-mass stars has yet to be realized. Many advantages of the infrared must however be acknowledged, like its strong potential for circumstellar material and atmosphere diagnostics, and its insensitivity to obscuration. This is important when one considers the typical distances one works to locate massive stars, often in the plane of our Galaxy. The use of infrared diagnostics is particularly relevant with regards to the first generation of stars, thought to be very massive.

This Special Session provided an opportunity to discuss the results obtained for massive stars from existing infrared facilities (VLTs/VLTI, Spitzer, Herschel, CRIRES, GAIA,...) as well as tools for interpreting infrared data (e.g. atmosphere modeling) and observing capabilities of future facilities (ELTs, JWST,...). It was split into three topics.

The first topic of this Special Session dealt with obscured and distant clusters. To improve the knowledge of the (rare) massive objects, the infrared domain is crucial as it reveals obscured and/or distant clusters, like those close to the Galactic Center. Such studies, by providing many new objects to work on, enable us to better understand the massive stars as a population and to reveal the strong impact of massive stars on the environment and clusters themselves, thanks to the eroding effect of their energetic radiation and dynamical interactions.

The Session continued with presentations on the determination of stellar and wind parameters of (mostly evolved) massive stars, which remain poorly constrained. However, new wind diagnostics extended to include infrared data are being developed. They provide additional leverage to select between competing wind models (including different clumping scenarios). Metallicity studies also benefit from access to infrared wavelengths, particularly with regards to tracing the chemistry of circumstellar environments.

Finally, the third topic of this Session considered matter ejection by massive stars (winds, LBV eruptions, supernovae). With the recent reduction in 201 cobserved $201 \mathrm{~d}$ mass-loss rates (by a factor of about 3), episodic matter ejection represents crucial, but poorly understood mechanisms needed for understanding the evolution of massive stars.

The SOC - Jura Borissova (Chile), Margaret Hanson (USA), Fabrice Martins (France), Paco Najarro (Spain), Yaël Nazé (Belgium, chair), Barbara Whitney (USA) 